\title{
SOX9-activated FARSA-AS1 predetermines cell growth, stemness, and metastasis in colorectal cancer through upregulating FARSA and SOX9
}

Taicheng Zhou', Lili Wu' ${ }^{2}$, Ning Ma', Fuxin Tang ${ }^{1}$, Zhuomin Yu' ${ }^{1}$, Zhipeng Jiang ${ }^{1}$, Yingru Li ${ }^{1}$, Zhen Zong ${ }^{3}$ and Kunpeng $\mathrm{Hu}^{4}$

\begin{abstract}
SRY-box transcription factors (SOXs) are effective inducers for the formation of stem-like phenotypes. As a member of SOX family, SOX9 (SRY-box transcription factor 9) has been reported to be highly expressed and exert oncogenic functions in multiple human cancers. In this study, we hypothesized that SOX9 could regulate the function of cancer stem/initiating cells (CSCs) to further facilitate the progression of colorectal cancer (CRC). Then, stable transfection of shRNAs was used to silence indicated genes. Loss-of-function experiments were conducted to demonstrate the in vitro function of CRC cells. In vivo study was conducted to determine the changes in tumorigenesis and metastasis in vivo. Bioinformatics analyses and mechanistic experiments were employed to explore the downstream molecules. Presently, GEPIA data indicated that SOX9 was upregulated in 275 COAD (colon adenocarcinoma) samples relative to 349 normal tissues. Besides, we also proved the upregulation of SOX9 in CRC cell lines (HCT15, SW480, SW1116, and HT-29) compared to normal NCM-460 cells. Silencing of SOX9 suppressed cell growth, stemness, migration, and invasion. Mechanistically, SOX9 activated the transcription of IncRNA phenylalanyl-tRNA synthetase subunit alpha antisense RNA 1 (FARSA-AS1), while FARSA-AS1 elevated SOX9 in turn by absorbing miR-18b-5p and augmented FARSA via sequestering miR-28-5p. Furthermore, loss of FARSA-AS1 hindered malignant phenotypes in vitro and blocked tumor growth and metastasis in vivo. Notably, we testified that FARSA-AS1 aggravated the malignancy in CRC by enhancing SOX9 and FARSA. Our study unveiled a mechanism of SOX9-FARSA-AS1-SOX9/FARSA loop in CRC, which provides some clews of promising targets for CRC.
\end{abstract}

\section{Introduction}

Colorectal cancer $(\mathrm{CRC})$ has been reported as one of the most malignant cancers in the world ${ }^{1,2}$. Because of the

\footnotetext{
Correspondence: Zhen Zong (zhenzo2922@163.com) or Kunpeng Hu (ku47477333@163.com)

${ }^{1}$ Department of Gastroenterological Surgery and Hernia Center, The Sixth Affiliated Hospital of Sun Yat-sen University, Guangdong Institute of Gastroenterology, Guangdong Provincial Key Laboratory of Colorectal and Pelvic Floor Diseases, Supported by National Key Clinical Discipline, 510655 Guangzhou, Guangdong, China

${ }^{2}$ Department of Medical Ultrasonics, Third Affiliated Hospital of Sun Yat-sen University, Guangdong Key Laboratory of Liver Disease Research, 510630 Guangzhou, Guangdong, China

Full list of author information is available at the end of the article These authors contributed equally: Taicheng Zhou, Lili Wu, Ning Ma Edited by S. Tait
}

high occurrence and death rate of CRC, people's health has been affected strictly. Even though there are much advance in the therapy of CRC, CRC still acts as a serious disease. It has been studied that various oncogenes participate in the progression of CRC, which provides novel targets for treating $\mathrm{CRC}^{3}$. Nevertheless, it is still unclear about the exact function and mechanism of oncogenes played in CRC.

As a member of SOX family, SRY-box transcription factor 9 (SOX9) can regulate cancer progression by affecting the transcription of diverse genes ${ }^{4,5}$. It has been reported that SOX9 is involved in the progression of prostate cancer and gastric cancer by regulating WNT signaling pathway ${ }^{6,7}$. Meanwhile, SOX9 can modulate cell

\section{(c) The Author(s) 2020}

(c) (i) Open Access This article is licensed under a Creative Commons Attribution 4.0 International License, which permits use, sharing, adaptation, distribution and reproduction cc) in any medium or format, as long as you give appropriate credit to the original author(s) and the source, provide a link to the Creative Commons license, and indicate if changes were made. The images or other third party material in this article are included in the article's Creative Commons license, unless indicated otherwise in a credit line to the material. If material is not included in the article's Creative Commons license and your intended use is not permitted by statutory regulation or exceeds the permitted use, you will need to obtain permission directly from the copyright holder. To view a copy of this license, visit http://creativecommons.org/licenses/by/4.0/. 
stemness in hepatocellular carcinoma ${ }^{8,9}$. Also, inhibited SOX9 hampers cell growth, migration, invasion, and EMT in thyroid cancer ${ }^{10}$. SOX9 can regulate the self-renewal of cancer stem cells in hepatocellular carcinoma ${ }^{11}$. However, the function of SOX9 in CRC still remains largely unknown. In this study, we are going to search the role and probable molecular mechanism of SOX9 in CRC.

With the development in sequencing technologies, it has been clearer that long noncoding RNAs (lncRNAs) are a novel group of RNA transcripts with more than 200 nucleotides in length ${ }^{12}$. More and more studies have proved that ncRNAs are dysregulated in cancers, and IncRNAs can participate in the regulation on biological behaviors of cancer cells, such as cell growth, apoptosis, migration, and invasion ${ }^{13,14}$. For example, lncRNA NEAT1 affects cell growth and apoptosis in $\mathrm{CRC}^{15}$. Also, lncRNA AB073614 regulates cell growth and metastasis by modulating PI3K/AKT pathway in $\mathrm{CRC}^{16}$. Besides, lncRNA TUG1 regulates the chemoresistance of CRC cells $^{17}$. Phenylalanyl-tRNA synthetase subunit alpha antisense RNA 1 (FARSA-AS1) is a newly found lncRNA that has been scarcely explored in CRC. In our study, we are going to explore the function and underlying molecular mechanism of FARSA-AS1 in CRC.

The competing endogenous RNA (ceRNA) network has recently been proposed, in which lncRNAs can function as microRNAs (miRNAs) sponges to regulate the expression of messenger RNAs (mRNAs) targeted by above miRNAs ${ }^{18,19}$. For example, lncRNA NORAD can function as a ceRNA to regulate EMT process and metastasis in pancreatic cancer ${ }^{20}$. Meanwhile, lncRNA H19 can play the role of ceRNA to modulate EMT process and metastasis in bladder cancer via sponging $m i R-29 b$ $3 p^{21}$. Also, IncRNA SNHG3 can be a ceRNA to promote the progression of $\mathrm{CRC}^{22}$. In our study, we searched the position of FARSA-AS1 in CRC cells and further explored the possible molecular mechanism of FARSA-AS1 in CRC.

\section{Materials and methods}

\section{Cell lines and culture}

Human CRC cell lines (HCT15, SW480, SW1116, and HT-29) and normal human colon epithelial cell line (NCM-460), all from ATCC (Manassas, VA), were allowed to propagate in the humidified incubator of $5 \%$ $\mathrm{CO}_{2}$ at $37^{\circ} \mathrm{C}$. DMEM medium (Gibco, Carlsbad, CA) was applied for culturing cells, with $1 \%$ Pen/Strep solution and $10 \%$ FBS (Gibco) as the supplements.

\section{Total RNA extraction and qRT-PCR}

Total cellular RNAs were extracted using Trizol based on the standard protocol. After that, the obtained RNAs were then used for cDNA synthesis with PrimeScript RT reagent kit (Takara, Otsu, Japan). Gene expression was quantified by qRT-PCR using Power SYBR Green
Table 1 The sequences of PCR primers.

\begin{tabular}{lll}
\hline Genes & Forward primer $\left(\mathbf{5}^{\prime} \mathbf{>} \mathbf{3}^{\prime}\right)$ & Reverse primer $\left(\mathbf{5}^{\prime}>\mathbf{3}^{\prime}\right)$ \\
\hline SOX9 & TCTGAACGAGAGCGAGAGC & CCGTTCTCACCGACTTCCT \\
NOS2 & CGCATGACCTGGTGTIGG & GCACATCCCCGCAAACATAG \\
FOS & CAAGCGGAGACAGACCAACT & GTGAGCTGCCAGGATGAACT \\
PVRL1 & ATTCCCCTACACCCCGTCTC & GGGGTACTGCAGGTTCTGTG \\
(NECTIN1) & & \\
FARSA-AS1 & TCCTGCTATCGCTTCCCAGT & GGTGGGACGTAATAGGAAGGT \\
GAPDH & GGAGCGAGATCCCTCCAAAAT & GGCTGTGTCATACTTCTCATGG \\
U6 & CTCGCTTCGGAGCACA & AACGCTTCACGAATTGCGT \\
FARSA & GCCCTCAAGCCCTACAACT & CTGGAAGAGGGGTCAAAGT \\
ALDH1A1 & CTGCCGGGAAAGCAATCTG & TCTTAGCCCGCTCAACACTC \\
CD133 & GTGGCGTGTGGGCTATGAC & CCAACTCCAACCATGAGGAAGACG \\
miR-18a- & GCGAGTAAGGTGCATCTAGT & CTCAACTGGTGTCGTGGA \\
$5 p$ & & \\
miR-18b- & GCGAGTAAGGTGCATCTAGT & CTCAACTGGTGTCGTGGA \\
$5 p$ & & \\
miR-28-5p & CGAGAAGGAGCTCACAGTCT & CTCAACTGGTGTCGTGGA \\
miR-708- & GCGAGAAGGAGCTACAATC & CTCAACTGGTGTCGTGA \\
$5 p$ & & \\
\hline
\end{tabular}

(TaKaRa) on ABI Prism 7900HT (Applied Biosystems, Foster City, CA, USA) and then calculated with $2^{-\Delta \Delta C t}$ method. GAPDH or $U 6$ acted as the internal reference for normalization. The primer sequences were shown in Table 1.

\section{Western blot}

Based on the instruction, total cellular protein extracts were acquired with RIPA lysis buffer, dissolved with $10 \%$ SDS-PAGE and then transferred to PVDF membranes. Followed by sealing with $5 \%$ skim milk, membranes were processed with primary antibodies against GAPDH (loading control) SOX9, and FARSA, and then with the secondary antibodies tagged with HRP subsequently. The antibodies were all purchased from Abcam (Cambridge, MA) and employed after dilution. Protein bands were visualized with the help of the enhanced chemiluminescence (ECL) fluorescence test kit (Amersham, Arlington Heights, IL).

\section{Transfection}

The specifically designed shRNAs and NC shRNAs were produced by Genepharma Company (Shanghai, China) for the depletion of SOX9 and FARSA-AS1 in SW480 and SW1116 cells. Besides, the pcDNA3.1-SOX9 and pcDNA3.1-FARSA were obtained through inserting corresponding cDNA sequences into pcDNA3.1 vectors (Invitrogen) for overexpressing SOX9 and FARSA, with the empty vector as negative controls. MiR-18b-5p/miR28-5p mimics and corresponding NC mimics (Genepharma) were constructed for overexpressing $m i R-18 b-5 p$ or $m i R-28-5 p$, respectively. Cell transfection was achieved using Lipofectamine 2000 (Invitrogen, Carlsbad, CA). Samples were collected after $48 \mathrm{~h}$ of transfection. 


\section{Colony formation assay}

The cultured cells at logarithmic growth were harvested and added to the six-well plates for the 14-day culture process at $37{ }^{\circ} \mathrm{C}$ in $5 \% \mathrm{CO}_{2}$. After washing by PBS and fixing with $4 \%$ paraformaldehyde, cells were processed with crystal violet solution for staining, followed by counting manually.

\section{EdU assay}

Cultured cells were placed at $5 \times 10^{4}$ cells/well to the 96-well plates for treating with EdU staining kit (Ribobio, Guangzhou, China). After that, cell nuclei were dualstained with DAPI solution, and then cells were subjected to final observation with fluorescence microscope (Olympus, Tokyo, Japan).

\section{Flow cytometer analysis of cell apoptosis}

On the basis of protocol, cell apoptosis was monitored with Annexin-V fluorescein isothiocyanate (FITC)/propidium iodide (PI) dual-staining kit (BD Biosciences, San Jose, CA). In brief, after treating with FITC-Annexin-V and PI in succession for $15 \mathrm{~min}$ in the dark, the apoptotic cells were analyzed using FACS cytometry (BD Biosciences).

\section{Sphere-formation assay and secondary and tertiary tumor sphere-formation assays}

SW480 and SW1116 cells were plated in 96-well ultralow attachment plates (Corning, New York, NY) and cultured in the sphere medium of DMEM with $10 \mathrm{ng} /$ mL EGF (Peprotech Inc., Rocky Hill, NJ, USA), $10 \mathrm{ng} / \mathrm{mL}$ bFGF (Peprotech Inc., Rocky Hill, NJ, USA), and 1×B27 (Invitrogen). After 1 week, the number of spheres was counted and the images were taken under an optical microscope (Olympus). In addition, the secondary and tertiary tumor sphere-formation assays were conducted according to the previous protocol ${ }^{23}$.

\section{Transwell assay}

The upper side of polycarbonate transwell filter (Corning) chamber was coated with Matrigel (BD Biosciences) for invasion assay and the chambers without Matrigel were prepared for migration analysis. Then, to the upper chambers were added with $5 \times 10^{4}$ cells in serum-free medium, while the complete medium in the lower chamber acted as the supplements. One day later, the migrating or invading cells in the bottom were processed with $0.5 \%$ crystal violet after fixing by $4 \%$ paraformaldehyde, followed by cell counting under the microscope.

\section{Luciferase reporter assays}

The fragments of FARSA-AS1 promoter with wild-type or mutant SOX9 binding sites were inserted into pGL3-basic vectors (Promega, Madison, WI). Then the recombinant constructs were subjected to the cotransfection with $S O X 9$ silencing plasmids into CRC cells. The pmirGLO-FARSA-AS1-WT/Mut and pmirGLO-SOX9 3'UTR-WT/Mut reporters were formed using the corresponding full-length sequences with wild-type or mutated $m i R-18 b-5 p$ binding sites, or pmirGLO-FARSA 3'UTR reporter with full-length FARSA 3'UTR sequence, were constructed and then cotransfected severally with miR-18b-5p mimics or NC mimics into CRC cells. Similarly, the pmirGLO-FARSA-AS1-WT/Mut and pmirGLO-FARSA-WT/Mut reporters were obtained through cloning corresponding full-length sequences covering wild-type or mutant miR-28-5p binding sites into pmirGLO vectors (Promega), and then were applied for cotransfecting with miR-28-5p mimics or NC mimics into CRC cells. The activities of firefly luciferase and Renilla luciferase were both tested by using dual luciferase reporter gene assay kit (BioTek, Winooski, VT) to determine the relative luciferase activity.

\section{TOP/FOP-flash assay}

TOP/FOP-flash assay was carried out as described previously $^{24}$. In short, cells were cotransfected with TOP/ FOP-Flash (Genechem) and different plasmids for $S O X 9$ overexpression, SOX9 inhibition, or FARSA-AS1 inhibition. After normalizing to the Renilla luciferase activity, the TOP/FOP ratio was measured via dual luciferase reporter system (Promega).

\section{ChIP assay}

One percent formalin was used to fix CRC cells to obtain DNA-protein cross-linking, followed by the cutting of DNA fragments into $200-500 \mathrm{bp}$ via ultrasonic. Cell lysates with indicated fragments were subjected to immunoprecipitation with SOX9 antibody or control IgG antibody (Millipore, Billerica, MA). Then the precipitated DNA fragments were captured by using magnetic beads. The qRT-PCR was employed for quantifying the precipitated DNA.

\section{Pull-down analyses}

For DNA pull-down, the 5' biotin-labeled FARSA-AS1 promoter and non-labeled promoter probes were conjugated with beads for culturing the protein extracts from CRC cells for $2 \mathrm{~h}$. Proteins were subjected to western blot analysis. For RNA pull-down, the wild-type and mutated miR-18b-5p fragments covering FARSA-AS1 or SOX9 interacting sequences were synthesized and biotinylated into Bio-miR-18b-5p-WT/Mut probes. The wild-type and mutated miR-28-5p fragments containing FARSA-AS1 or FARSA interacting sequences were acquired and biotinylated into Bio-miR-28-5p-WT/Mut probes. After incubation with cell extracts, qRT-PCR was carried out to detect indicated RNAs. 
FISH

The specific RNA FISH probe of FARSA-AS1 was synthesized by Bersinbio Company (Guangzhou, China) and applied for FISH assay as required by supplier. After DAPI dual-staining, cells were imaged under fluorescence microscope.

\section{IF staining}

To detect SOX9 localization in CRC cells, IF assay was implemented with specific SOX9 antibody purchased from Abcam. Simply put, cells were fixed with $4 \%$ paraformaldehyde for $10 \mathrm{~min}$, permeabilized via $0.1 \%$ Triton X-100 for 5 min and then sealed with $1 \%$ BSA for $1 \mathrm{~h}$. Afterwards, cells were then incubated overnight with SOX9 antibodies at $4{ }^{\circ} \mathrm{C}$, followed by the incubation for $1 \mathrm{~h}$ with the AlexaFluor ${ }^{\circledR} 488$ secondary antibodies at room temperature. Following staining via DAPI, cells were observed under a fluorescence microscope.

\section{Subcellular fractionation}

The separation of cell nucleus and cytoplasm was achieved with the PARIS Kit (Invitrogen) on the basis of standard method. Then, the level of indicated RNAs (FARSA-AS1, GAPDH, or U6) in different fractions was analyzed via qRT-PCR, with GAPDH and $U 6$ acted as cytoplasmic and nuclear indicators, respectively.

\section{Animal experiment}

Six weeks old of female BALB/c nude mice (SLRC Laboratory Animal Center, Shanghai, China) were commercially acquired for animal study, with the approval of the Institutional Animal Care and Use Committee of the Sixth Affiliated Hospital of Sun Yat-sen University. Each mouse was injected subcutaneously with $3 \times 10^{6}$ CRC cells that were transfected with sh-NC or sh-FARSA-AS1 (three mice each group), with tumor volume measured every 4 days. Four weeks later, mice were sacrificed, and then the xenografts were collected for weight mensuration and qRT-PCR analysis. For in vivo metastasis analysis, $1 \times$ $10^{7} \mathrm{CRC}$ cells transfected with sh-NC or sh-FARSA-AS1 were injected into the tail vein of mice. Six weeks later, the lungs of mice (total three lungs each group) were collected for counting the pulmonary metastatic nodules. The lungs fixed in formalin were processed with paraffin embedding for Hematoxylin and Eosin (H\&E) staining.

\section{Statistical analysis}

Data from independent bio-triplications were shown as mean \pm standard deviation (SD) and analyzed via Graphpad Prism 6 software. The comparison of differences between groups was appropriately achieved by $t$-test (twotailed) and one-way or two-way ANOVA, as per the significant level at $p<0.05$.

\section{Results}

SOX9 is upregulated in CRC cells and promotes cell growth, stemness, migration, and invasion

According to the data from GEPIA (http://gepia.cancerpku.cn/), we found that SOX9 was upregulated in colon adenocarcinoma (COAD) tissues (Fig. 1A). Thereafter, we planned to detect the expression of SOX9 in CRC cell lines (HCT15, SW480, SW1116, and HT-29) and human normal colonic epithelial cell (NCM-460). Before that, we identified that no SOX9 mutation was found in SW1116 and HT-29 cells but four kinds of SOX9 mutations were predicted in HCT15 cells by using COSMIC tool (http:// cancer.sanger.ac.uk/cancergenome/projects/cell_lines/). Besides, a previous study also suggested no SOX9 mutation in SW480 cell line ${ }^{25}$. Then the results of qRT-PCR showed the high levels of SOX9 mRNA and protein in three CRC cell lines with wild-type SOX9, especially in SW480 and SW1116 cells (Fig. 1B). Afterwards, we knocked down SOX9 expression in SW480 and SW1116 cells for further study (Fig. 1C). Data from colony formation assay and EdU assay indicated that loss of SOX9 inhibited the proliferative capacity of SW480 and SW1116 cells (Fig. 1D, E). In contrast, the apoptosis of SW480 and SW1116 cells was induced by SOX9 silencing (Fig. 1F). Importantly, we found that the efficiency of sphere formation by SW480 and SW1116 cells was restrained and the size of spheres was diminished under the inhibition of SOX9 (Fig. 1G). To further explore the impact of SOX9 on CRC cell stemness, we conducted secondary and tertiary tumor sphere-formation assays. Results manifested that the absence of SOX9 hindered the formation of secondary and tertiary tumor spheres from CRC cells (Fig. S1A). Consistently, we also discovered the reduced levels of $A L D H$ and CD133 in CRC cells with SOX9 depletion (Fig. S1B). Furthermore, the outcomes of transwell assay unveiled that the migration and invasion capacities were also hampered in SOX9 silenced SW480 and SW1116 cells (Fig. 1H, I). To further know the influence of SOX9 on the malignant behaviors of colorectal cells, we performed gain-offunction assays in the normal NCM-460 cells. It was proved that upregulating SOX9 in NCM-460 cells facilitated proliferation, enhanced stemness and accelerated migration and invasion (Fig. S1C-I). Besides, considering the importance of SOX9 in regulating $\mathrm{Wnt} / \beta$-catenin pathway, we then tested its impact on this pathway in CRC. As expected, SOX9 deficiency dramatically lowered the activity of $\mathrm{Wnt} / \beta$-catenin signaling in SW480 and SW1116 cells, whereas its overexpression evidently fortified the activity of this pathway in NCM-460 cells (Fig. S1J). These data suggested that SOX9 is upregulated in CRC cells and it promotes oncogenic phenotypes in CRC cells by activating $\mathrm{Wnt} / \beta$-catenin pathway. 


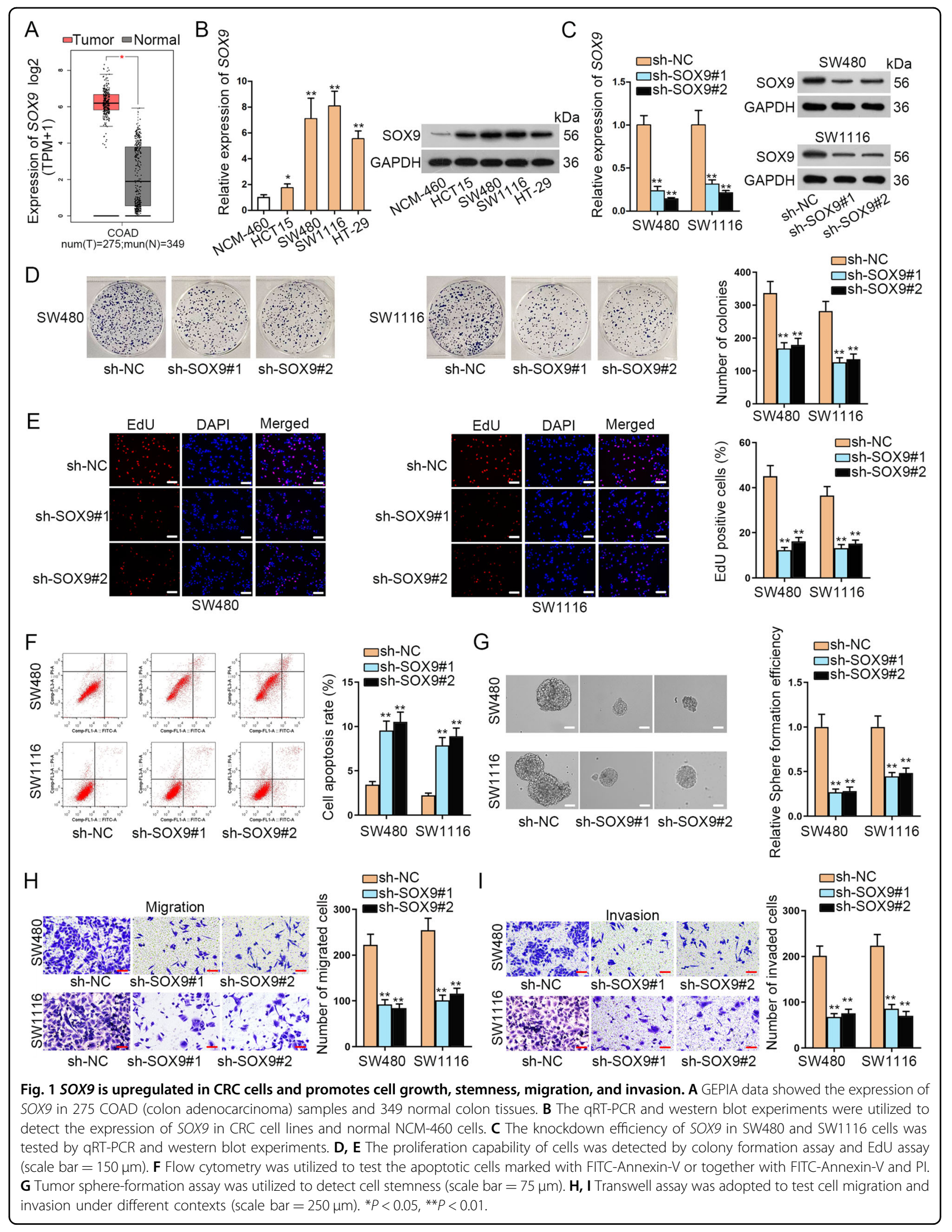




\section{SOX9 binds to FARSA-AS1 promoter to elevate FARSA-AS1 in CRC cells}

Then, we researched the similar genes of SOX9 in COAD samples from GEPIA (http://gepia.cancer-pku.cn/). As shown in Fig. 2A, top 20 genes correlated with SOX9 in COAD samples were selected and the results of qRT-PCR indicated that four (NOS2, FOS, PVRL1, and FARSA-AS1) among the 20 genes presented upregulation in SW1116 cells compared to NCM-460 cells. Then, we detected the expression of these four genes in CRC cells with or without SOX9 depletion, and results displayed that FARSA-AS1 was significantly downregulated in SOX9 silenced SW480 and SW1116 cells (Fig. 2B). In addition, the expression of FARSA-AS1 was found to be upregulated in CRC cells
(Fig. 2C). Subsequently, we overexpressed SOX9 in SW480 and SW1116 cells (Fig. S2A), resulting in the upregulation of FARSA-AS1 in such cells (Fig. 2D). These data indicated that $S O X 9$ could positively regulated FARSA-AS1. It was reported that SOX9 could function as a transcription factor and activate gene expression by binding to the promoter of genes ${ }^{26}$. Here, we also discovered that a major proportion of SOX9 located in the nucleus of CRC cells (Fig. S2B), further highlighting the potential for SOX9 to affect FARSA-AS1 transcription. To confirm this, we used UCSC (http://genome.ucsc.edu/) and JASPAR (http:// jaspar.genereg.net/) websites to obtain the DNA motif and the binding site of SOX9 on FARSA-AS1 promoter (Fig. 2E, F). Luciferase reporter assay results revealed that

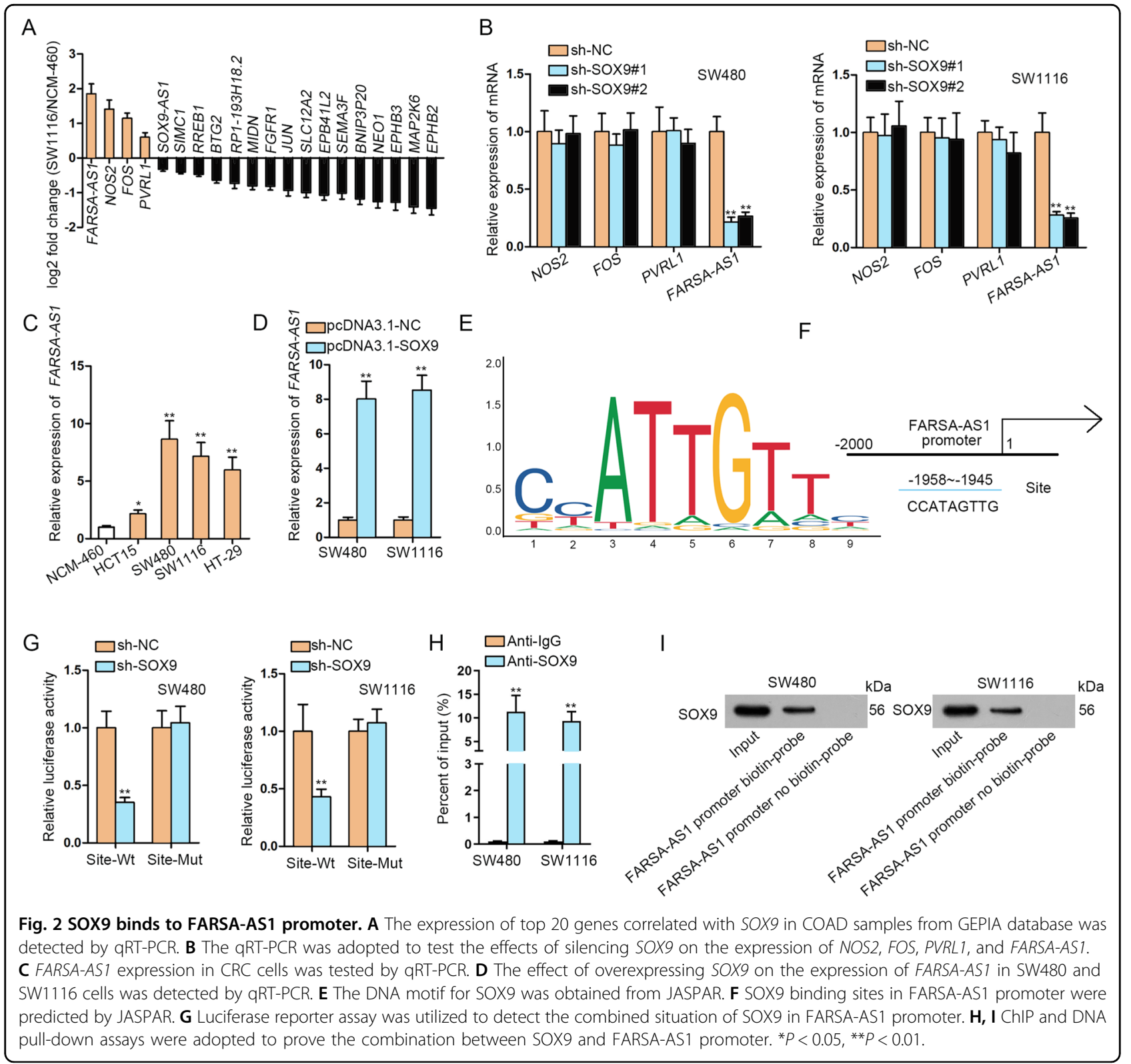


SOX9 silencing decreased the luciferase activity of wildtype FARSA-AS1 promoter, whereas that of FARSA-AS1 promoter with mutant binding site displayed no significant changes (Fig. 2G). Data from ChIP and DNA pull-down assays further confirmed the interactivity of SOX9 with FARSA-AS1 promoter (Fig. 2H, I). Conclusively, SOX9transcriptionally activates FARSA-AS1 in CRC cells.

\section{FARSA-AS1 facilitates the malignant processes in CRC cells}

To explore the role of FARSA-AS1 in CRC, we stably silenced FARSA-AS1 expression in SW480 and SW1116 cells by transfecting with sh-FARSA-AS1\#1/2 (Fig. 3A). As a result, silencing FARSA-AS1 also led to declined activity of Wnt/ $\beta$-catenin pathway in both CRC cells (Fig. S2C). Moreover, FARSA-AS1 depletion remarkably suppressed the proliferation of SW480 and SW1116 cells (Fig. 3B, C). Flow cytometry elucidated the promoted apoptosis in FARSA-AS1 downregulated SW480 and SW1116 cells (Fig. 3D). Importantly, knockdown of FARSA-AS1 markedly repressed the ability of SW480 and SW1116 cells to form primary, secondary, and tertiary spheres (Fig. 3E and Fig. S2D). Also, the levels of $A L D H$ and $C D 133$ were reduced in CRC cells in response to FARSA-AS1 absence (Fig. S2E). Similarly, the inhibited migration and invasion were observed in SW480 and SW1116 cells with FARSA-AS1 deficiency (Fig. 3F, G). In sum, FARSA-AS1 facilitates CRC cell growth, stemness, migration, and invasion.

\section{FARSA-AS1 upregulates SOX9 by sponging miR-18b-5p}

Interestingly, we then discovered that the levels of SOX9 mRNA and protein were both reduced along with FARSAAS1 downregulation in SW480 and SW1116 cells (Fig. 4A). To explore the mechanism of FARSA-AS1 in regulating $S O X 9$, we tested the localization of FARSA-AS1 in SW480 and SW1116 cells via FISH and subcellular fractionation assays. The results demonstrated that FARSA-AS1 was mainly distributed in the cytoplasm of both CRC cells (Fig. 4B, C), which suggested the regulation of FARSA-AS1 on SOX9 at post-transcriptional level. It has been reported that lncRNAs could regulate the expression of mRNAs by functioning as a 'miRNA Sponge' ${ }^{\text {19,27 }}$. Later, we aimed to find out the potential miRNAs that could simultaneously bind to FARSA-AS1 and SOX9. In this case, 45 miRNAs were predicted for FARSA-AS1 through DIANA (http://diana.imis.athena-innovation.gr/ DianaTools/index.php? $r=$ site/page\&view $=$ software), and 197 miRNAs were predicted for SOX9 via starBase (http:// starbase.sysu.edu.cn/index.php), with only two miRNAs (miR-18a-5p and $m i R-18 b-5 p)$ shared between them (Fig. 4D). Further, only miR-18b-5p was validated to be under-expressed in CRC cells in comparison to NCM-460 cells (Fig. 4E). Then, the binding sequences between $m i R$ $18 b-5 p$ and FARSA-AS1 or SOX9 were shown (Fig. 4F).
Before verifying the interaction between them, we ascertained that $m i R-18 b-5 p$ expression was increased in CRC cells after transfecting with miR-18b-5p mimics (Fig. S2F). As validated by the data from luciferase reporter assay, enhanced expression of miR-18b-5p lessened the luciferase activity of FARSA-AS1-WT but not that of FARSAAS1-Mut (Fig. 4G). In addition, RNA pull-down assay results confirmed the specific interaction of FARSA-AS1 with miR-18b-5p-WT rather than miR-18b-5p-Mut (Fig. 4H). Meanwhile, the combination between $m i R-18 b-5 p$ and SOX9 was testified similarly by such manners (Fig. 4I, J). Taken all together, FARSA-AS1 upregulates SOX9 in CRC by sponging $m i R-18 b-5 p$.

\section{SOX9 fully rescues the effects of FARSA-AS1 on CRC cells}

To the purpose of verifying whether the regulation of FARSA-AS1 on CRC progression was mediated by $S O X 9$, some restoration assays were carried out. According to the outcomes of colony formation and EdU assays, we found that SOX9 overexpression fully rescued the inhibitory effect of FARSA-AS1 silencing on CRC cell proliferation (Fig. 5A, B and Fig. S3A, B). The induced apoptosis in FARSA-AS1 depleted cells was also fully recovered by upregulated SOX9 (Fig. $5 \mathrm{C}$ and Fig. S3C). FARSA-AS1 knockdown inhibited the sphere-formation efficiency of SW480 and SW1116 cells, whereas the cotransfection of pcDNA3.1-SOX9 fully counteracted this effect (Fig. 5D and Fig. S3D). Meanwhile, the suppressed ability of CRC cells to form secondary and tertiary spheres was completely recovered due to SOX9 upregulation (Fig. S3E), so were the restrained levels of $A L D H$ and CD133 (Fig. S3F). In addition, ectopic expression of SOX9 fully countervailed FARSA-AS1 deficiency-mediated suppression on SW480 and SW1116 cell migration and invasion (Fig. 5E, F and Fig. S3G, H). All the results indicated that $S O X 9$ fully rescues the effects of FARSAAS1 on the phenotypes of CRC cells.

\section{FARSA-AS1 upregulates FARSA via sequestering miR-28-5p}

Through UCSC (http://genome.ucsc.edu/), FARSA, the nearby gene of FARSA-AS1, was found. It has been reported that lncRNAs could exert functions in cancer by regulating their nearby genes ${ }^{28}$. Therefore, we wondered the influence of FARSA-AS1 on FARSA expression in CRC cells. As illustrated in Fig. 6A, FARSA mRNA and protein expressions were significantly decreased upon FARSAAS1 knockdown. Besides, we found that FARSA mRNA and protein levels were highly expressed in CRC cells in contrast to normal NCM-460 cells (Fig. 6B, C). Interestingly, luciferase reporter assay results displayed that the luciferase activity of FARSA 3'UTR presented no evident differences in cells with miR-18b-5p upregulation (Fig. S4A). In addition, $m i R-18 b-5 p$ overexpression did not affect FARSA mRNA and protein levels in both 


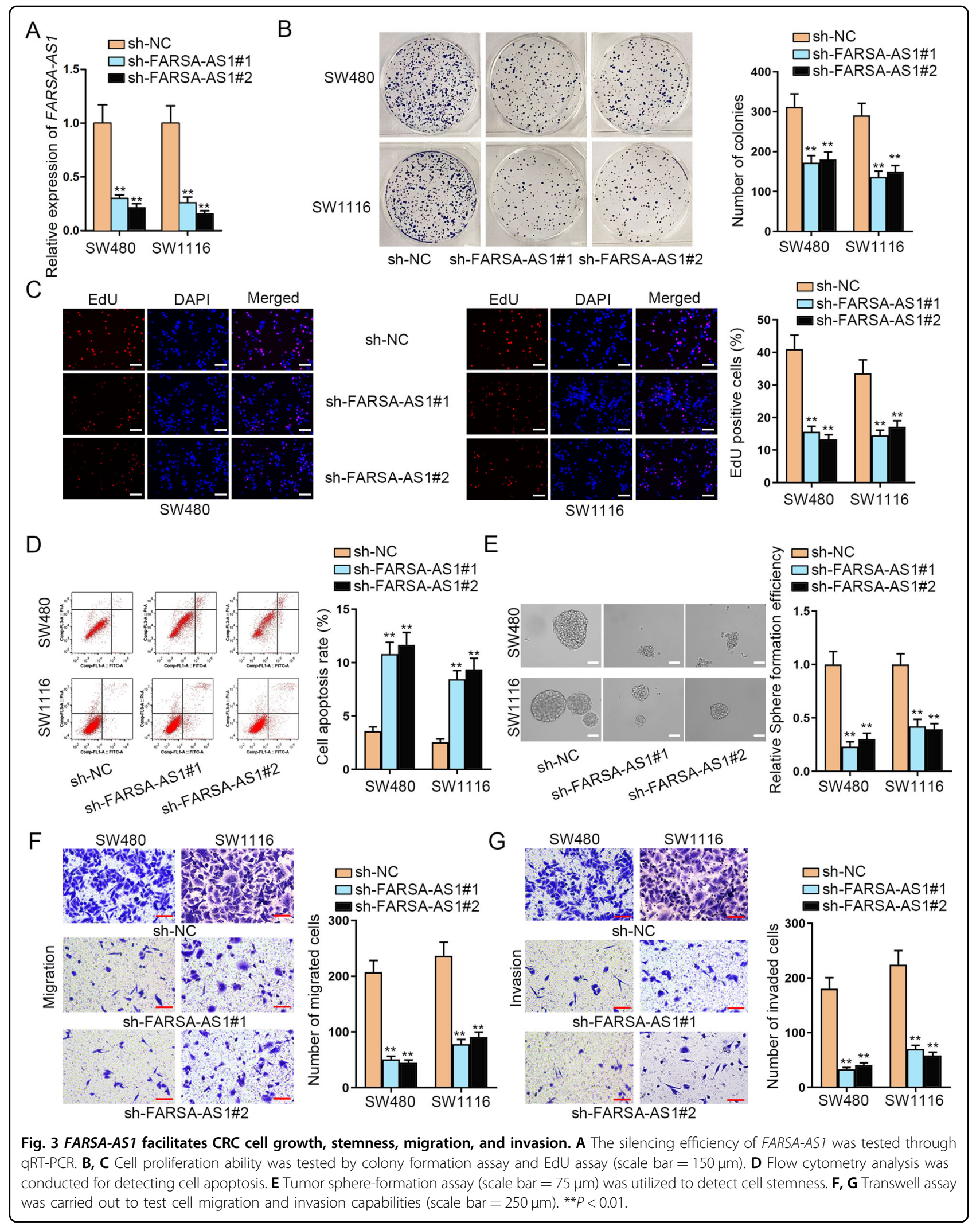




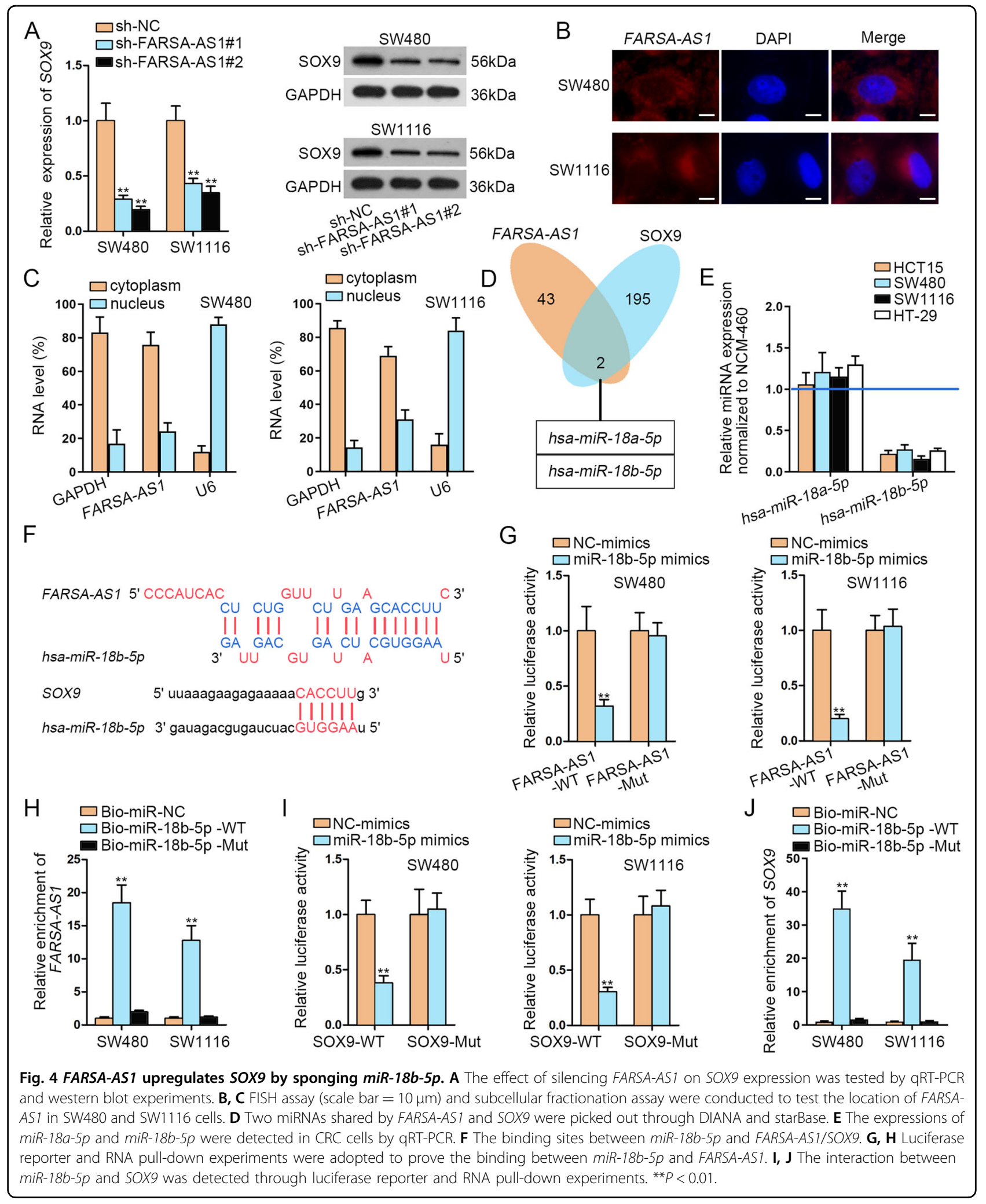

CRC cells (Fig. S4B). These data revealed that FARSA-AS1 regulated $F A R S A$ expression not by sponging $m i R-18 b-5 p$. In consequence, we searched for miRNAs that combined with FARSA-AS1 and FARSA, and then miR-28-5p and miR-708-5p were screened out (Fig. 6D). Furthermore, the results of qRT-PCR manifested that $m i R-28-5 p$ was 


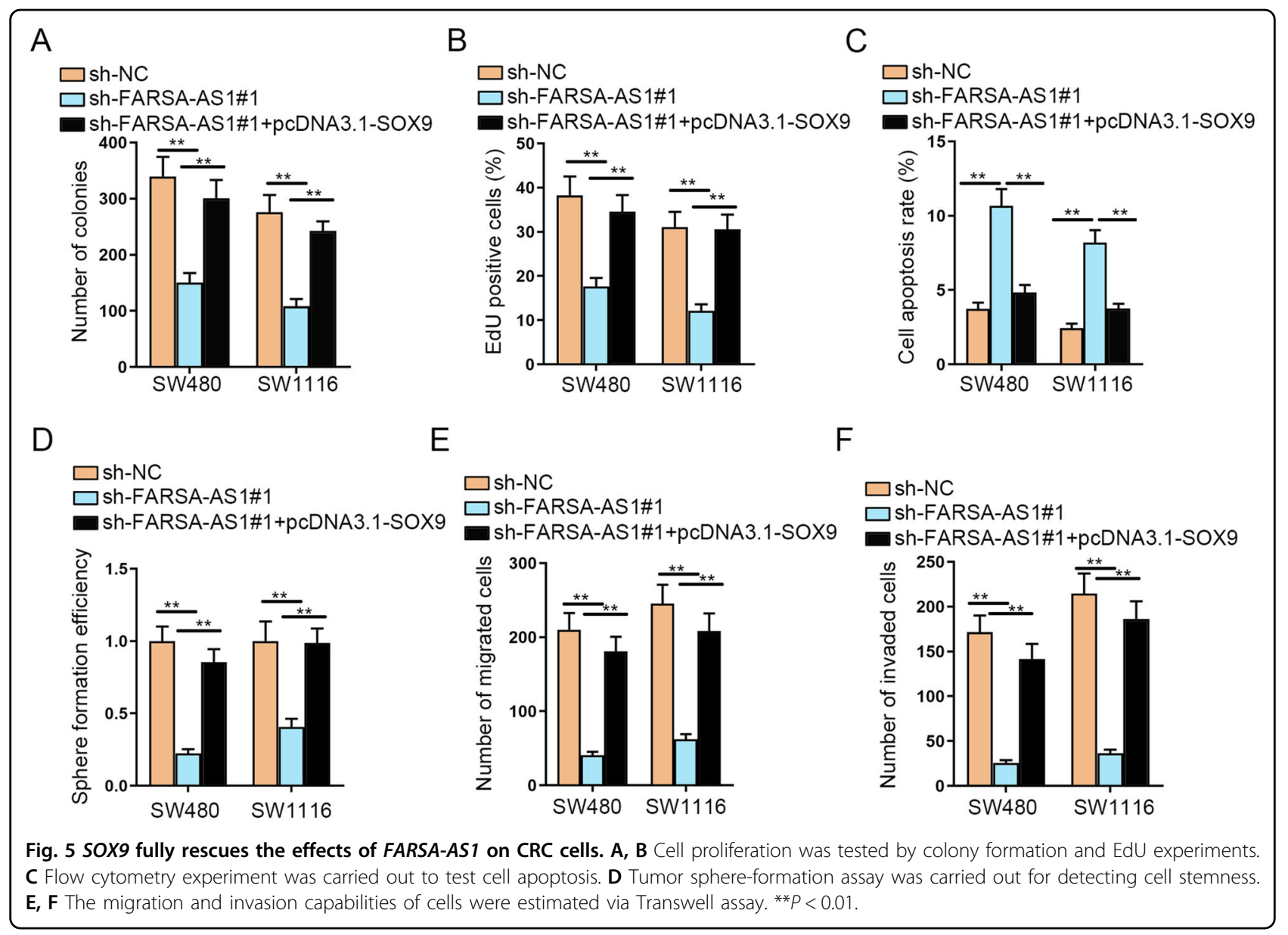

downregulated in CRC cells, while miR-708-5p expression showed no notable changes (Fig. 6E). Hence, we speculated that miR-28-5p might be implicated in the regulation of FARSA-AS1 on FARSA in CRC. The predicted binding sequences between $m i R-28-5 p$ and $F A R S A-A S 1$ or FARSA were illustrated in Fig. 6F. Then, we overexpressed $m i R-28-5 p$ in SW480 and SW1116 cells (Fig. 6G). As anticipated, the luciferase activity of FARSA-AS1-WT was considerably diminished with the transfection of miR-28$5 p$ mimics, while miR-28-5p overexpression did not impact the luciferase activity of FARSA-AS1-Mut (Fig. 6H). RNA pull-down assay data implied the remarkable enrichment of FARSA-AS1 specially by biotinylated miR-28-5p-WT (Fig. 6I). Furthermore, the interaction between $m i R-28-5 p$ and FARSA was also validated by luciferase reporter assay and RNA pull-down assay (Fig. 6J, K). All data suggested that FARSA-AS1 upregulates FARSA via sequestering $m i R-28-5 p$.

\section{FARSA-AS1 enhances SOX9 and FARSA expressions to promote tumor progression in CRC}

To determine whether FARSA-AS1 functions in CRC by targeting $S O X 9$ and FARSA, a series of rescue experiments were designed and conducted. At first, we tested the overexpression efficiency of pcDNA3.1-FARSA in CRC cells, and results illustrated that FARSA expression was remarkably increased by pcDNA3.1-FARSA (Fig. 7A). As a result, overexpressed FARSA partially rescued the suppressed proliferation in FARSA-AS1 depleted cells, whereas the cotransfection of pcDNA3.1-FARSA and pcDNA3.1-SOX9 countervailed the repressive effect of FARSA-AS1 silencing on cell proliferation (Fig. 7B, C and Fig. S4C, D). FARSA-AS1 knockdown promoted the apoptosis of CRC cells, but this impact was partially recovered by FARSA upregulation, and mostly offset with the cotransfection of pcDNA3.1-FARSA and pcDNA3.1SOX9 (Fig. 7D and Fig. S4E). The hampered stemness of FARSA-AS1 silenced SW480 and SW1116 cells was partially reversed by transfecting with pcDNA3.1-FARSA, while restored upon the co-effect of FARSA overexpression and SOX9 upregulation (Fig. 7E and Fig. S4F, G). Also, the alleviated cell migration and invasion caused by silenced FARSA-AS1 was partially recovered by pcDNA3.1-FARSA transfection, but normalized in response to the co-upregulation of FARSA and SOX9 (Fig. 7F, G and Fig. S4H). In the meantime, we also 


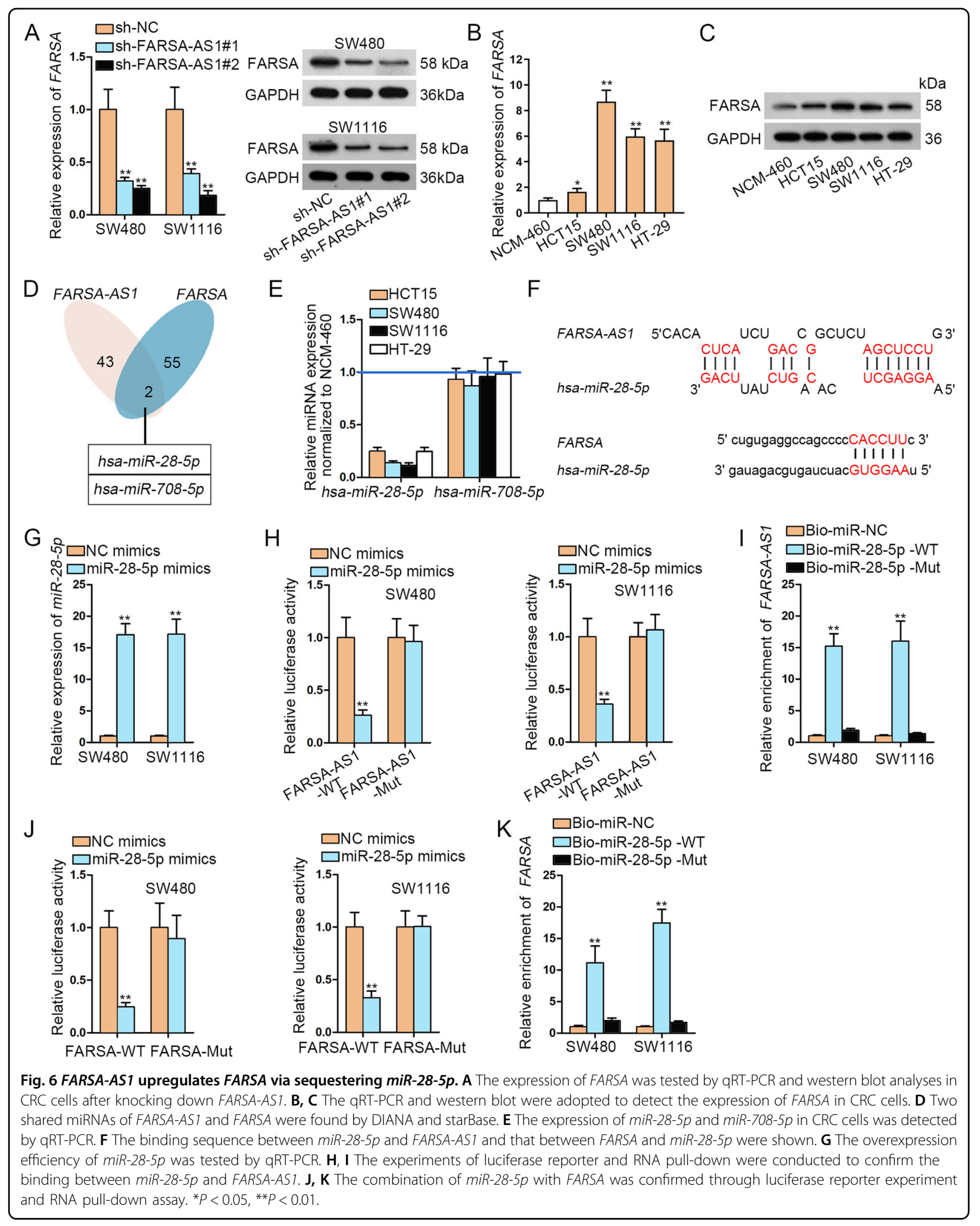




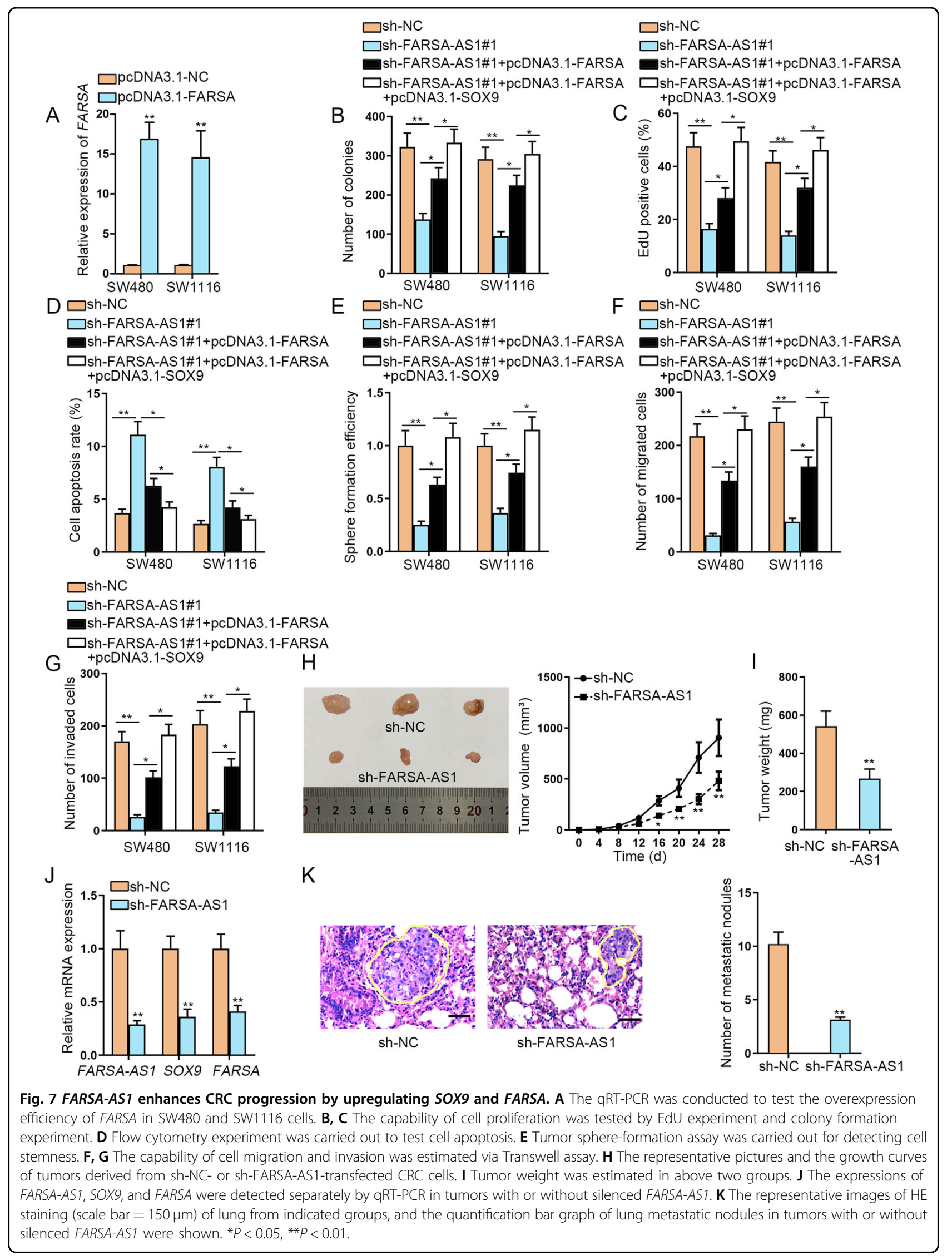


conducted in vivo assays to further verify the importance of FARSA-AS1 for CRC progression. As expected, the tumors from FARSA-AS1-silenced CRC cells looked smaller owing to the slower growth rate compared with those from sh-NC group (Fig. 7H). Resultantly, the weight of tumors in sh-FARSA-AS1 group was also lighter than that in control group (Fig. 7I). Further, we certified the reduced levels of FARSA-AS1, SOX9 and FARSA in tumors derived from CRC cells with silenced FARSA-AS1 (Fig. 7J). Moreover, the results of in vivo tumor metastasis model showed that the number of lung metastatic nodules was remarkably decreased in mice injected with FARSAAS1-silenced CRC cells (Fig. 7K). In conclusion, FARSA$A S 1$ contributes to CRC progression by upregulating SOX9 and FARSA.

\section{Discussion}

As a common malignancy, the incidence and mortality of $\mathrm{CRC}$ are increasing worldwide ${ }^{29}$. In spite of the research over years, we have not fully elucidated the mechanism underlying CRC tumorigenesis. Until now, we have not identified the effective diagnostic methods. Most patients with CRC are diagnosed at the middle or advanced stage and miss the chance to be timely treated ${ }^{30}$. Therefore, it is in an urgent need to explore the potential biomarkers for the treatment of CRC. Here, we searched TGCA database and found a SOX9 presented a high level in CRC tissues. In various cancer types, SOX9 has been observed to be deregulated because of the amplification and other mechanisms ${ }^{31,32}$. Recent researches showed that the upregulation of SOX9 predicted unsatisfactory survival rate in hepatocellular carcinoma ${ }^{33}$ and gastric cancer ${ }^{6}$. In our study, the results demonstrated that $S O X 9$ was remarkably upregulated in CRC cells, and SOX9 knockdown suppressed cell proliferation, stemness, migration, and invasion. SOX9 was also reported as a transcription factor to activate the expression of some genes $^{34}$. Here, we found that silencing SOX9 decreased the expression of IncRNA FARSA-AS1, and SOX9 overexpression increased FARSA-AS1 expression. In addition, SOX9 was also confirmed to combine with FARSA-AS1 promoter. These data manifested that SOX9 is an oncogene in CRC and functions as a transcription activator of FARSA-AS1 in CRC cells.

In recent years, the critical effects of lncRNAs have attracted the attentions of many researchers for the reason of their abnormal regulation on tumor progression. In particular, an increasing number of lncRNAs have been identified as biomarkers in cancers ${ }^{35}$. Although a series of IncRNAs have been found to be dysregulated in multiple cancers, it is still needed to investigate the molecular mechanism of lncRNAs in cancers. As is widely reported, lncRNAs are associated with the progression of diverse cancers, including CRC. For example, LINC01296 contributes to CRC progression via PI3K/AKT pathway and targeting miR-26a/GALNT3 axis $^{36}$. YAP1-mediated MALAT1 enhances CRC angiogenesis and epithelialmesenchymal transition via blocking miR-126-5 $p^{37}$. FARSA-AS1 is a newly identified lncRNA, which has not been researched in CRC. In this study, FARSA-AS1 was found to be highly expressed in CRC cells. Silencing FARSA-AS1 inhibited cell proliferation, stemness, migration, and invasion. All results suggested that FARSA-AS1 presented a carcinogenic property in CRC.

MicroRNAs (miRNAs) are identified as small noncoding RNAs with about 22 nucleotides in length. MiRNAs have been revealed to play important parts in regulating the biological behaviors of cancer cells. For instance, miR$200 \mathrm{c} / 141$ targets to HIPK1/ $\beta$-catenin axis to regulate the heterogeneity of breast cancer stem cells ${ }^{38}$. MiR-4516 functions as a novel oncogene in glioblastoma and predicts poor prognosis via targeting PTPN14 ${ }^{39}$. MiR-371a-3p targets to $T O B 1$ and facilitates cell proliferation and invasion in gastric cancer ${ }^{40}$. Generally, lncRNAs regulate the expression of mRNAs by secluding miRNAs. Therefore, miRNAs are of great importance in ceRNA network ${ }^{41}$. MiR-18b-5p was reported in lung adenocarcinoma ${ }^{42}$, whereas its role and molecular mechanism remain largely unclear in CRC. In this study, we found that FARSA-AS1 regulated $S O X 9$ by sponging $m i R-18 b-5 p$. Restoration assay manifested that SOX9 upregulation fully rescued the inhibitive effects of FARSA-AS1 silencing on the functions of CRC cells. Then, we found that FARSA-AS1 could also modulate its nearby gene, FARSA, through sponging $m i R$ 28-5p. According to the results of rescue experiments, the impacts of FARSA-AS1 downregulated cells were partially counteracted by FARSA upregulation, but mostly recovered upon the co-overexpression of FARSA and SOX9.

In conclusion, FARSA-AS1 was upregulated in CRC cells and facilitated CRC progression by upregulating SOX9 and FARSA. This discovery provides a helpful theoretical basis for the exploration of effective CRC therapeutic strategies.

\section{Acknowledgements}

We appreciate the supports of our experimenters.

\section{Author details \\ ${ }^{1}$ Department of Gastroenterological Surgery and Hernia Center, The Sixth Affiliated Hospital of Sun Yat-sen University, Guangdong Institute of Gastroenterology, Guangdong Provincial Key Laboratory of Colorectal and Pelvic Floor Diseases, Supported by National Key Clinical Discipline, 510655 Guangzhou, Guangdong, China. ${ }^{2}$ Department of Medical Ultrasonics, Third Affiliated Hospital of Sun Yat-sen University, Guangdong Key Laboratory of Liver Disease Research, 510630 Guangzhou, Guangdong, China. ${ }^{3}$ Department of Gastroenterological Surgery, The Second Affiliated Hospital of Nanchang University, No.1 Mingde Road, 330006 Nanchang, Jiangxi, China. ${ }^{4}$ Department of General Surgery, The Third Affiliated Hospital of Sun Yat-sen University, No.2693 Kaichuang Road, Huangpu, 510000 Guangzhou, Guangdong, China}

Conflict of interest

The authors declare that they have no conflict of interest. 


\section{Publisher's note}

Springer Nature remains neutral with regard to jurisdictional claims in published maps and institutional affiliations.

Supplementary Information accompanies this paper at (https://doi.org/ 10.1038/s41419-020-03273-4).

Received: 15 February 2020 Revised: 13 November 2020 Accepted: 17 November 2020

Published online: 14 December 2020

\section{References}

1. Booth, R. A. Minimally invasive biomarkers for detection and staging of colorectal cancer. Cancer Lett. 249, 87-96 (2007).

2. Jemal, A. et al. Global cancer statistics. CA Cancer J. Clin. 61, 69-90 (2011).

3. Arends, M. J. Pathways of colorectal carcinogenesis. Appl. Immunohistochem. Mol. Morphol. 21, 97-102 (2013).

4. Foster, J. W. et al. Campomelic dysplasia and autosomal sex reversal caused by mutations in an SRY-related gene. Nature 372, 525-530 (1994).

5. Wagner, T. et al. Autosomal sex reversal and campomelic dysplasia are caused by mutations in and around the SRY-related gene SOX9. Cell 79, 1111-1120 (1994).

6. Santos, J. C. et al. SOX9 elevation acts with canonical WNT signaling to drive gastric cancer progression. Cancer Res. 76, 6735-6746 (2016).

7. Ma, F. et al. SOX9 drives WNT pathway activation in prostate cancer. J. Clin. Invest. 126, 1745-1758 (2016).

8. Kawai, T. et al. SOX9 is a novel cancer stem cell marker surrogated by osteopontin in human hepatocellular carcinoma. Sci. Rep. 6, 30489 (2016).

9. Richtig, G. et al. SOX9 is a proliferation and stem cell factor in hepatocellular carcinoma and possess widespread prognostic significance in different cancer types. PLOS ONE 12, e0187814 (2017).

10. Huang, J. \& Guo, L. Knockdown of SOX9 inhibits the proliferation, invasion, and EMT in thyroid cancer cells. Oncol. Res. 25, 167-176 (2017).

11. Liu, C. et al. Sox9 regulates self-renewal and tumorigenicity by promoting symmetrical cell division of cancer stem cells in hepatocellular carcinoma. Hepatology 64, 117-129 (2016).

12. Gutschner, T. \& Diederichs, S. The hallmarks of cancer: a long non-coding RNA point of view. RNA Biol. 9, 703-719 (2012)

13. Saxena, A. \& Carninci, P. Long non-coding RNA modifies chromatin: epigenetic silencing by long non-coding RNAs. Bioessays 33, 830-839 (2011).

14. Wang, K. C. \& Chang, H. Y. Molecular mechanisms of long noncoding RNAs. Mol. Cell 43, 904-914 (2011).

15. Peng, W., Wang, Z. \& Fan, H. LncRNA NEAT1 impacts cell proliferation and apoptosis of colorectal cancer via regulation of Akt signaling. Pathol. Oncol. Res. 23, 651-656 (2017).

16. Wang, $Y$. et al. LncRNA AB073614 regulates proliferation and metastasis of colorectal cancer cells via the PI3KJAKT signaling pathway. Biomed. Pharmacother. 93, 1230-1237 (2017).

17. Li, C., Gao, Y., Li, Y. \& Ding, D. TUG1 mediates methotrexate resistance in colorectal cancer via miR-186/CPEB2 axis. Biochem. Biophys. Res. Commun. 491, 552-557 (2017).

18. Cesana, M. et al. A long noncoding RNA controls muscle differentiation by functioning as a competing endogenous RNA. Cell 147, 358-369 (2011).

19. Tay, Y., Rinn, J. \& Pandolfi, P. P. The multilayered complexity of ceRNA crosstalk and competition. Nature 505, 344-352 (2014).

20. $\mathrm{Li}, \mathrm{H}$. et al. Long noncoding RNA NORAD, a novel competing endogenous RNA, enhances the hypoxia-induced epithelial-mesenchymal transition to promote metastasis in pancreatic cancer. Mol. Cancer 16 169 (2017).

21. LV, M. et al. IncRNA H19 regulates epithelial-mesenchymal transition and metastasis of bladder cancer by miR-29b-3p as competing endogenous RNA. Biochim. Biophys. Acta Mol. Cell Res. 1864, 1887-1899 (2017).

22. Huang, W. et al. The long non-coding RNA SNHG3 functions as a competing endogenous RNA to promote malignant development of colorectal cancer. Oncol. Rep. 38, 1402-1410 (2017).

23. Serrano-Oviedo, L. et al. Identification of a stemness-related gene panel associated with BET inhibition in triple negative breast cancer. Cell Oncol. (Dordr.) 43, 431-444 (2020).

24. Zheng, $A$. et al. Long non-coding RNA LUCAT1/miR-5582-3p/TCF7L2 axis regulates breast cancer stemness via Wnt/ß-catenin pathway. J. Exp. Clin. Cancer Res. 38, 305-305 (2019).

25. Prévostel, C. et al. SOX9 is an atypical intestinal tumor suppressor controlling the oncogenic Wnt/ß-catenin signaling. Oncotarget 7, 82228-82243 (2016).

26. Wang, L. et al. SOX9/miR-203a axis drives PI3KAKT signaling to promote esophageal cancer progression. Cancer Lett. 468, 14-26 (2019).

27. Karreth, F. A. \& Pandolfi, P. P. ceRNA cross-talk in cancer: when ce-bling rivalries go awry. Cancer Discov. 3, 1113-1121 (2013).

28. Qian, W. et al. IncRNA ZEB1-AS1 promotes pulmonary fibrosis through ZEB1mediated epithelial-mesenchymal transition by competitively binding miR141-3p. Cell Death Dis. 10, 129 (2019).

29. Siegel, R. L., Miller, K. D. \& Jemal, A. Cancer statistics, 2018. CA Cancer J. Clin. 68 7-30 (2018)

30. $\mathrm{Yu}$, J. et al. Metagenomic analysis of faecal microbiome as a tool towards targeted non-invasive biomarkers for colorectal cancer. Gut 66, 70-78 (2017).

31. Wang, L. et al. Linc-ROR promotes esophageal squamous cell carcinoma progression through the derepression of SOX9. J. Exp. Clin. Cancer Res. 36, 182 (2017).

32. Wang, L. et al. Unbalanced YAP-SOX9 circuit drives stemness and malignant progression in esophageal squamous cell carcinoma. Oncogene $\mathbf{3 8}$ 2042-2055 (2019).

33. Leung, C. O. et al. Sox 9 confers stemness properties in hepatocellular carcinoma through Frizzled-7 mediated Wnt/beta-catenin signaling. Oncotarget 7, 29371-29386 (2016)

34. Gubbay, J. et al. A gene mapping to the sex-determining region of the mouse $Y$ chromosome is a member of a novel family of embryonically expressed genes. Nature 346, 245-250 (1990).

35. Chandra Gupta, S. \& Nandan Tripathi, Y. Potential of long non-coding RNAs in cancer patients: From biomarkers to therapeutic targets. Int. J. Cancer $\mathbf{1 4 0}$ 1955-1967 (2017).

36. Liu, B. et al. Correction to: LINC01296/miR-26a/GALNT3 axis contributes to colorectal cancer progression by regulating O-glycosylated MUC1 via PI3K AKT pathway. J. Exp. Clin. Cancer Res. 38, 367 (2019).

37. Sun, Z. et al. YAP1-induced MALAT1 promotes epithelial-mesenchymal transition and angiogenesis by sponging miR-126-5p in colorectal cancer. Oncogene 38, 2627-2644 (2019).

38. Liu, B. et al. miR-200c/141 regulates breast cancer stem cell heterogeneity via targeting HIPK1/beta-catenin axis. Theranostics 8, 5801-5813 (2018).

39. Cui, T. et al. miR-4516 predicts poor prognosis and functions as a novel oncogene via targeting PTPN14 in human glioblastoma. Oncogene $\mathbf{3 8}$, 2923-2936 (2019)

40. Guo, $H$. et al. MicroRNA-371a-3p promotes progression of gastric cancer by targeting TOB1. Cancer Lett. 443, 179-188 (2019).

41. Tan, J. Y. et al. Extensive microRNA-mediated crosstalk between IncRNAs and mRNAs in mouse embryonic stem cells. Genome Res. 25, 655-666 (2015).

42. Xue, M. et al. IncRNA ZFPM2-AS1 promotes proliferation via miR-18b-5p/ VMA21 axis in lung adenocarcinoma. J. Cell. Biochem. 121, 313-321 (2019). 
